\title{
Analysis of the Expression and Clinical Significance of VIPR1 in Lung Adenocarcinoma Based on TCGA Database
}

\section{Xiaobo Li ( 954930360@qq.com )}

Zhengzhou University First Affiliated Hospital https://orcid.org/0000-0002-2407-5504

\section{Yunfei Wang}

Zhengzhou University First Affiliated Hospital

\section{Xiaowan Wei}

Zhengzhou University First Affiliated Hospital

\section{Guojun Zhang}

Zhengzhou University First Affiliated Hospital

\section{Huaqi Wang}

Zhengzhou University First Affiliated Hospital

\section{Research Article}

Keywords: lung adenocarcinoma, VIPR1, immune infiltration, MDSC

Posted Date: November 19th, 2021

DOI: https://doi.org/10.21203/rs.3.rs-1058170/v1

License: (c) (1) This work is licensed under a Creative Commons Attribution 4.0 International License. Read Full License 


\section{Abstract}

Background: The expression of VIPR1 is associated with the prognosis of many malignant tumors. To analyze the expression and clinical significance of vasoactive intestinal peptide Types I (VIPR1) in lung adenocarcinoma based on The Cancer Genome Atlas (TCGA)database.

Methods and results囚The RNASeq data, clinical data and prognosis data of lung adenocarcinoma and normal lung tissue were downloaded from TCGA database. The expression difference of VIPR1 mRNA in lung adenocarcinoma and normal lung tissue $\bigotimes p<0.05 \bigotimes$, and the correlations of the expression levels of VIPR1 in lung adenocarcinoma with clinical pathological characteristics and prognosis were analyzed. The possible regulatory signaling pathways of VIPR1 were predicted by the gene set enrichment analysis (GSEA). CIBERSORT was used to analyze the expression of immune cells in tumor tissues and normal tissues. TIMER was used to analyze the relationship between VIPR1 and immune cell expression. The expression levels of VIPR1 mRNA in lung adenocarcinoma tissues were significantly lower than that in normal lung tissues. The expression levels of VIPR1 mRNA were significantly correlated with gender, $T$ staging $\otimes \mathrm{N}$ staging and pathological grade $\otimes p<0.05 \bigotimes$ but not with age, $\mathrm{M}$ staging and survival status. Survival analysis showed that the survival time of patients with low expression of VIPR1 was significantly lower than that of patients with high expression. In addition, the expression level of VIPR1 in lung adenocarcinoma was negatively correlated with the infiltration level of myeloid inhibitory cells $(r=$ $-0.448, p<0.01$ ). Through GSEA functional enrichment analysis, it was found that VIPR1 was mainly related to cell cycle pathway, p53 signal pathway, DNA replication pathway, RNA degradation pathway and so on.

Conclusions $₫ \mathrm{VIPR} 1$ gene may be a new target for the clinical prognosis and targeted therapy of lung adenocarcinoma.

\section{Background:}

vasoactive intestinal peptide (VIP), as a vasoactive intestinal peptide, participates in smooth muscle relaxation of lung and intestinal epithelium[1], which can promote the survival of peripheral neurons and inhibit neuronal death after injury[2]. It can inhibit the proliferation and metastasis of human renal cell carcinoma A498 cells by up-regulating p53 and down-regulating the expression of NF-KB, VEGF and CD34 [3]. At the same time, it also has immunomodulatory function and is a specific chemokine and activator of macrophages, $B$ cells and T cells [4]. It can also inhibit the production of many proinflammatory factors and chemokines, including TNF- $a$, IL-6, IL-12, MIP-1 and MIP-2, and promote the production of some antagonists such as anti-inflammatory cytokines such as IL-10 and IL-1R [3]. Among them, TNF- $a$, IL-12, MIP-1, MIP-2 and IL-10 are mainly regulated by VIPR1 [5]. VIPR1 is the most important receptor for VIP, which is mainly found in some regions of the human brain. It is also expressed in rat and human pulmonary macrophages, pulmonary venous vascular smooth muscle and airway epithelium from bronchioles to respiratory bronchioles [6]. The expression of VIPR1 was different in different tumor tissues, and the expression level in breast cancer was 5 times higher than that in normal breast tissue [7], 
but lower in hepatocellular carcinoma [8], thyroid cancer [9], gastric cancer [10] and non-small cell lung cancer [11, 12]. In breast cancer, VIP can induce cell proliferation by acting on VIPR1 [13], while in hepatocellular carcinoma, patients with high expression of VIPR1 have a relatively good prognosis [8]. More and more studies have proved that VIPR1 plays an important role in the differentiation and treatment of human cancer, such as antagonizing the binding of VIP and VIPR1 in prostate cancer, inhibiting tumor cell growth [8], functional imaging of tumor tissue and targeted chemotherapy in breast cancer[14]. Up-regulation of VIPR1 expression in lung adenocarcinoma can inhibit the growth of H1299 cells $[15,16]$. In recent years, immunotherapy has increasingly become a hot spot in the treatment of lung cancer, and its effect is also related to immune cell infiltration in the tumor microenvironment. At present, there are no studies on the role of VIPR1 in immune infiltration of lung adenocarcinoma, so this study intends to use a variety of bioinformatics methods to study the role of VIPR1 in lung adenocarcinoma, and explore its effect on the pattern of immune cell infiltration, so as to provide clinical prognostic markers of new immune-related genes.

\section{Materials And Methods:}

\section{Data collection, collation and analysis:}

The transcriptional group data and related clinical data of lung adenocarcinoma patients were downloaded from TCGA database (https://portal.gdc.cancer.gov/)). The data download format was standardized FPKM format, of which 497 cases were tumor samples and 54 cases were normal control samples. To analyze the difference of gene expression between tumor tissue and normal tissue, and to explore the relationship between VIPR1 expression level and total survival time of patients with lung adenocarcinoma by univariate Cox regression analysis. T staging, $\mathrm{M}$ staging, $\mathrm{N}$ staging, age, gender and pathological grade were selected as the clinicopathological features of lung adenocarcinoma. The box chart was used to show the difference of gene expression between normal group and tumor group, and the difference was statistically significant $(p<0.05)$.

\section{The Human Protein Atlas:}

Analysis of the distribution of VIPR1 in normal lung tissue and lung adenocarcinoma tissue (scale: $50 \mu$ m) based on THE HUMAN PROTEIN ATLAS database (HPA, https://www.proteinatlas.org/).

\section{Gene enrichment analysis}

GSEA4.0.1 software is used to analyze and explore the important signal pathways related to VIPR1. According to the expression level of VIPR1, they were divided into high expression group and low expression group. Get the c2.cp.kegg.v7.4.symbols.gmt dataset through the GSEA website. The default weighted enrichment statistics method was used for enrichment analysis and the number of random combinations was set to 1000 . 


\section{Immune infiltration assessment:}

CIBERSORT (http://cibersort.stanford.edu/)) was used to estimate the composition of 22 kinds of immune cells in lung adenocarcinoma and normal lung tissues. Through deconvolution algorithm, CIBERSORT software can calculate the composition of immune cells in complex cells based on standardized gene expression data.

\section{Analysis of the relationship between VIPR1 and immune infiltrating cells:}

The relationship between VIPR1 expression and immune cell infiltration such as CD $4+T$ cells, B cells, neutrophils and myeloid inhibitory cells was analyzed by Immune plate of TIMER2.0 database (http://timer.cistrome.org/).

\section{Statistical analysis}

The R software (version 4.1.0) was used for data analysis. The expression of VIPR1 in lung adenocarcinoma and normal lung tissues was compared by wilcoxon rank sum test, the expression of VIPR1 in different TNM stages of lung adenocarcinoma was compared by one-way ANOVA, and the correlation between the two groups was tested by spearman correlation test. The relationship between VIPR1 and total survival time (OS) was analyzed by Kmuri M curve and tested by log-rank method. The test level is $a=0.05$.

\section{Results:}

\section{Relationship between VIPR1 gene expression level and clinical characteristics of patients with lung adenocarcinoma}

The clinical and transcriptional data of patients with lung adenocarcinoma were downloaded from TCGA public database. After analysis, we found that there was differential expression of VIPR1 in lung adenocarcinoma and normal lung tissues, and the expression in normal lung tissue (figure 1.K) was higher than that in lung adenocarcinoma tissue (figure 1.M). We continued to analyze the relationship between the high and low expression of VIPR1 and the prognosis of patients. The results showed that the patients with high expression of VIPR1 had a better prognosis than those with low expression (figure. 1. F). Further analysis showed that the expression of VIPR1 was correlated with gender, T staging, $\mathrm{N}$ staging and clinical grade (figure $1 . D, G, H, J$ ), but not with age, survival status and $M$ staging. 


\section{Functional enrichment Analysis of VIPR1}

The results of GSEA enrichment analysis showed that the samples with low expression of VIPR1 were significantly enriched in cell cycle pathway, p53 signal pathway, DNA replication pathway and RNA degradation pathway (figure 2 , table 1 ).

Table 1

Functional enrichment analysis of VIPR1 gene

\begin{tabular}{|c|c|c|c|c|}
\hline NAME & SIZE & & NES & NOM p-val \\
\hline KEGG_ALDOSTERONE_REGULATED_SODIUM_REABSORPTION & 42 & 0.5655199 & 1.99602 & 0 \\
\hline KEGG_CELL_CYCLE & 124 & -0.757659 & -2.37 & \\
\hline KEGG_ALZHEIMERS_DISEASE & 165 & -0.645564 & -2.2276 & 0 \\
\hline KEGG_PROTEASOME & 46 & -0.890569 & -2.2286 & \\
\hline GG_PYRIMIDINE_METABOLISM & 97 & -0.626209 & -2.1845 & 0.001949318 \\
\hline GG_PATHOGENIC_ESCHERICHIA_COLI_INFECTION & 56 & -0.654081 & -2.167 & \\
\hline KEGG_OOCYTE_MEIOSIS & 112 & -0.583873 & -2.1869 & \\
\hline KEGG_RNA_DEGRADATION & 59 & -0.641009 & -2.1709 & \\
\hline KEGG_PROTEIN_EXPORT & 24 & -0.797611 & -2.1206 & \\
\hline NS_DIS & 180 & -0.6 & -2.0782 & 0.002105263 \\
\hline & 28 & -0.7 & -2.0784 & \\
\hline SCRIP & 35 & -0.656776 & -2.0484 & 0.003913894 \\
\hline GG_UBIQUITIN_MEDIATED_PROTEOLYSIS & 134 & -0.519087 & -2.0372 & \\
\hline GG_PURINE_MÉTABOLISM & 156 & -0.497453 & -2.0228 & 0.001912046 \\
\hline DISEASE & 127 & -0.7 & -2.0111 & 0.004106776 \\
\hline & 36 & -0.8 & -2 & \\
\hline SPHATE_PATHWAY & 27 & 0321 & -1.9653 & 0.00212766 \\
\hline H_REPAIR & 23 & -0.779243 & -1.9494 & 0.003891051 \\
\hline KEGG_PROGESTERONE_MEDIATED_OOCYTE_MATURATION & 85 & -0.511501 & -1.9324 & \\
\hline KEGG_CITRATE_CYCLE_TCA_CYCLE & 31 & -0.732301 & -1.9498 & 0.001992032 \\
\hline KEGG_SPLICEOSOME & 127 & -0.645736 & -1.9536 & 0.001926782 \\
\hline & 68 & -0.5 & -1.9107 & 865672 \\
\hline METABOLISM & 15 & 3668 & -1.9005 & 0.004048583 \\
\hline GG_RNA_POLYMERASE & 28 & -0.658956 & -1.9025 & 0.003846154 \\
\hline KEGG_CYSTEINE_AND_METHIONINE_METABOLISM & 34 & -0.58691 & -1.8713 & 0.002 \\
\hline KEGG_AMYOTROPHIC_LATERAL_SCLEROSIS_ALS & 53 & -0.48055 & -1.7782 & 0.00408998 \\
\hline KEGG_N_GLYCAN_BIOSYNTHESIS & 46 & -0.523136 & -1.7458 & 0.025896415 \\
\hline KEGG_BASE_EXCISION_REPAIR & 35 & -0.628301 & -1.7196 & 0.01372549 \\
\hline
\end{tabular}

There is a difference in the expression of immune cells between lung adenocarcinoma and normal tissues

As shown in figure 3 , The proportion of immature B cells, plasma cells, activated memory CD4+T cells, follicular helper T cells, regulatory T cells, M1 macrophages and resting dendritic cells in lung 
adenocarcinoma tissues was higher than that in normal lung tissues. Normal lung tissue is rich in inactivated memory CD4+T cells, inactivated NK cells, monocytes, M0 macrophages, M2 macrophages, activated dendritic cells, resting mast cells, neutrophils, but no immature CD4+T cells were found.

There is a correlation between the expression level of VIPR1 gene and the expression of immune infiltrating cells

After TIMER2.0 analysis, we found that the epigenetic expression of VIPR1 motifs was negatively correlated with tumor-related fibrillar cells (Rho $=-0.103, p<0.01)$ and myeloid inhibitory cells (Rho=-0.488, $p<0.01$ ) (figure. 4).

\section{Discussion:}

The prevention, diagnosis and treatment of lung cancer is still a difficult problem today. Through the analysis of the TCGA database, we found that the expression of VIPR1 was low in lung adenocarcinoma, while the patients with high expression had a better prognosis and the research of others is also consistent[15]. VIPR1 is the main receptor of VIP. VIP can inhibit the production of TNF- $a$, IL-6 and VFGF[17]. Some studies have shown that PGE2, TNF- $a$, IL-6, IL-1 and VEGF combined with GM-CSF can induce CD33+ monocytes isolated from healthy subjects to differentiate into MDSC[18]. Myeloid inhibitory cells (MDSC) are developed from myeloid progenitor cells (CMP). They are mainly divided into two categories: mononuclear myeloid suppressor cells (M-MDSC) and polymorphonuclear myeloid suppressor cells (PMN-MDSC) $[19,20]$. Inhibitory immune infiltration microenvironment is one of the main reasons for tumor cell immune escape and poor efficacy of immunotherapy. MDSC is one of the main cells that constitute inhibitory immune infiltration microenvironment, which can inhibit the role of $T$ cells, B cells, macrophages and dendritic cells, and directly act on epithelial cells to promote their growth and mutation[17]. PMN-MDSC can produce ROS, which can directly destroy the binding of T cell TCR to MHC, while M-MDSC can consume T cells' important nutrients, such as L-arginine, thus inhibiting the function of T cells. It has been reported that inhibition of MDCS cell infiltration can inhibit the progression of lung cancer [21]. Tumor-associated fibroblasts (CAF) are the most abundant stromal cells in the tumor microenvironment, which can secrete a variety of chemokines to promote tumor cell growth, invasion and proliferation [22]. High infiltration of MDSC and CAF in tumor tissue often indicates a poor prognosis.

In this study, by analyzing the download data of TCGA database, we found that VIPR1 was low-expressed in lung adenocarcinoma, which suggested that VIPR1 may be an important tumor suppressor gene in lung adenocarcinoma. Further analysis of the relationship between VIPR1 and clinical characteristics of lung adenocarcinoma showed that the expression of VIPR1 was significantly correlated with gender, T staging, $\mathrm{N}$ staging and clinical grade. At the same time, the survival curve showed that the patients with high expression of VIPR1 had a better prognosis. These results suggest that the low expression of VIPR1 may be an unfavorable factor for the prognosis of lung adenocarcinoma. In order to further explore the mechanism of VIPR1 in lung adenocarcinoma, we found that the high expression of VIPR1 can inhibit the 
invasion of MDSC and CAF in tumor tissue by TIMER analysis. GSEA enrichment analysis showed that the samples with low expression of VIPR1 were mainly enriched in cell cycle, P53 signal pathway, DNA replication pathway and RNA degradation pathway. Therefore, we speculate that low expression of VIPR1 may promote the development of lung adenocarcinoma mainly by regulating cell cycle, P53 signal, DNA and RNA replication and degradation to promote MDSC and CAF infiltration. Therefore, we speculate that the high expression of VIPR1 may improve the prognosis of patients by inhibiting the production of MDSC.

\section{Conclusion}

VIPR1 gene may become a clinical prognostic index and a new target for targeted therapy of lung adenocarcinoma.

\section{Declarations}

\section{Acknowledgements}

Not applicable

\section{Author contributions}

GJZ and HQW conceived and designed the experiments. XBL and YFW analyzed the data. XBL and XWW wrote the manuscript.

\section{Funding}

This study is supported by the project of National Natural Science Foundation of China(81972182).

\section{Availability of data}

All data generated or analyzed during the present study are included in this published article.

\section{Competing interests}

The authors declare that they have no competing interests.

\section{Research involving human and/or animal participants}


This article does not contain any studies with human participants or animals performed by any of the authors.

\section{Ethical approval}

This article does not contain any studies with human participants performed by any of the authors.

\section{References}

1. Virgolini I, Raderer M, Kurtaran A, Angelberger P, Yang Q, Radosavljevic M, Leimer M, Kaserer K, Li SR, Kornek G, Hubsch P, Niederle B, Pidlich J, Scheithauer W (1996) Valent, 123I-vasoactive intestinal peptide (VIP) receptor scanning: update of imaging results in patients with adenocarcinomas and endocrine tumors of the gastrointestinal tract. Nucl Med Biol 23:685-692

2. Dufes C, Alleaume C, Montoni A, Olivier JC, Muller JM (2003) Effects of the vasoactive intestinal peptide (VIP) and related peptides on glioblastoma cell growth in vitro. J Mol Neurosci 21:91-102

3. Szliter EA, Lighvani S, Barrett RP, Hazlett LD (2007) Vasoactive intestinal peptide balances pro- and anti-inflammatory cytokines in the Pseudomonas aeruginosa-infected cornea and protects against corneal perforation. J Immunol 178:1105-1114

4. Reubi JC (2000) In vitro evaluation of VIP/PACAP receptors in healthy and diseased human tissues. Clinical implications. Ann N Y Acad Sci 921:1-25

5. Leceta J, Gomariz RP, Martinez C, Abad C, Ganea D, Delgado M, Ann (2000) N Y Acad Sci 921:92102

6. Derand R, Montoni A, Bulteau-Pignoux L, Janet T, Moreau B, Muller JM, Becq F (2004) Activation of VPAC1 receptors by VIP and PACAP-27 in human bronchial epithelial cells induces CFTR-dependent chloride secretion. Br J Pharmacol 141:698-708

7. Dagar S, Krishnadas A, Rubinstein I, Blend MJ, Onyuksel H (2003) VIP grafted sterically stabilized liposomes for targeted imaging of breast cancer: in vivo studies. J Control Release 91:123-133

8. Lu S, Lu H, Jin R, Mo Z (2019) Promoter methylation and H3K27 deacetylation regulate the transcription of VIPR1 in hepatocellular carcinoma. Biochem Biophys Res Commun 509:301-305

9. Suteau V, Seegers V, Munier M, Ben Boubaker R, Reyes C, Gentien D, Wery M, Croue A, Illouz F, Hamy A, Rodien P, Briet C (2021) G Protein-coupled Receptors in Radioiodine-refractory Thyroid Cancer in the Era of Precision Medicine. J Clin Endocrinol Metab 106:2221-2232

10. Li GH, Qian W, Song GQ, Hou XH (2007) Effect of vasoactive intestinal peptide on gastric adenocarcinoma. J Gastroenterol Hepatol 22:1328-1335

11. Moody TW, Walters J, Casibang M, Zia F, Gozes Y (2000) VPAC1 receptors and lung cancer. Ann N Y Acad Sci 921:26-32

12. Wang L, Sun Y, Jiang M, Zhang S, Wolfl S (2009) FOS proliferating network construction in early colorectal cancer (CRC) based on integrative significant function cluster and inferring analysis. 
Cancer Invest 27:816-824

13. Valdehita A, Bajo AM, Schally AV, Varga JL, Carmena MJ, Prieto JC (2009) Vasoactive intestinal peptide (VIP) induces transactivation of EGFR and HER2 in human breast cancer cells. Mol Cell Endocrinol 302:41-48

14. Dagar S, Sekosan M, Lee BS, Rubinstein I, Onyuksel H (2001) VIP receptors as molecular targets of breast cancer: implications for targeted imaging and drug delivery. J Control Release 74:129-134

15. Zhao L, Yu Z, Zhao B (2019) Mechanism of VIPR1 gene regulating human lung adenocarcinoma H1299 cells. Med Oncol 36:91

16. Kadara H, Fujimoto J, Yoo SY, Maki Y, Gower AC, Kabbout M, Garcia MM, Chow CW, Chu Z, Mendoza G, Shen L, Kalhor N, Hong WK, Moran C, Wang J, Spira A, Coombes KR (2014) Wistuba, II, Transcriptomic architecture of the adjacent airway field cancerization in non-small cell lung cancer. $J$ Natl Cancer Inst 106:dju004

17. Liu Y, Wei G, Cheng WA, Dong Z, Sun H, Lee VY, Cha SC, Smith DL, Kwak LW, Qin H (2018) Targeting myeloid-derived suppressor cells for cancer immunotherapy. Cancer Immunol Immunother 67:11811195

18. Ibanez-Vea M, Zuazo M, Gato M, Arasanz H, Fernandez-Hinojal G, Escors D, Kochan G (2018) Myeloid-Derived Suppressor Cells in the Tumor Microenvironment: Current Knowledge and Future Perspectives. Arch Immunol Ther Exp (Warsz) 66:113-123

19. Cassetta L, Baekkevold ES, Brandau S, Bujko A, Cassatella MA, Dorhoi A, Krieg C, Lin A, Lore K, Marini O, Pollard JW, Roussel M, Scapini P, Umansky V, Adema GJ (2019) Deciphering myeloid-derived suppressor cells: isolation and markers in humans, mice and non-human primates. Cancer Immunol Immunother 68:687-697

20. Safarzadeh E, Orangi M, Mohammadi H, Babaie F, Baradaran B (2018) Myeloid-derived suppressor cells: Important contributors to tumor progression and metastasis. J Cell Physiol 233:3024-3036

21. Sivagnanalingam U, Beatty PL, Finn OJ (2020) Myeloid derived suppressor cells in cancer, premalignancy and inflammation: A roadmap to cancer immunoprevention. Mol Carcinog 59:852861

22. Ishii G, Ochiai A, Neri S (2016) Phenotypic and functional heterogeneity of cancer-associated fibroblast within the tumor microenvironment. Adv Drug Deliv Rev 99:186-196

\section{Figures}



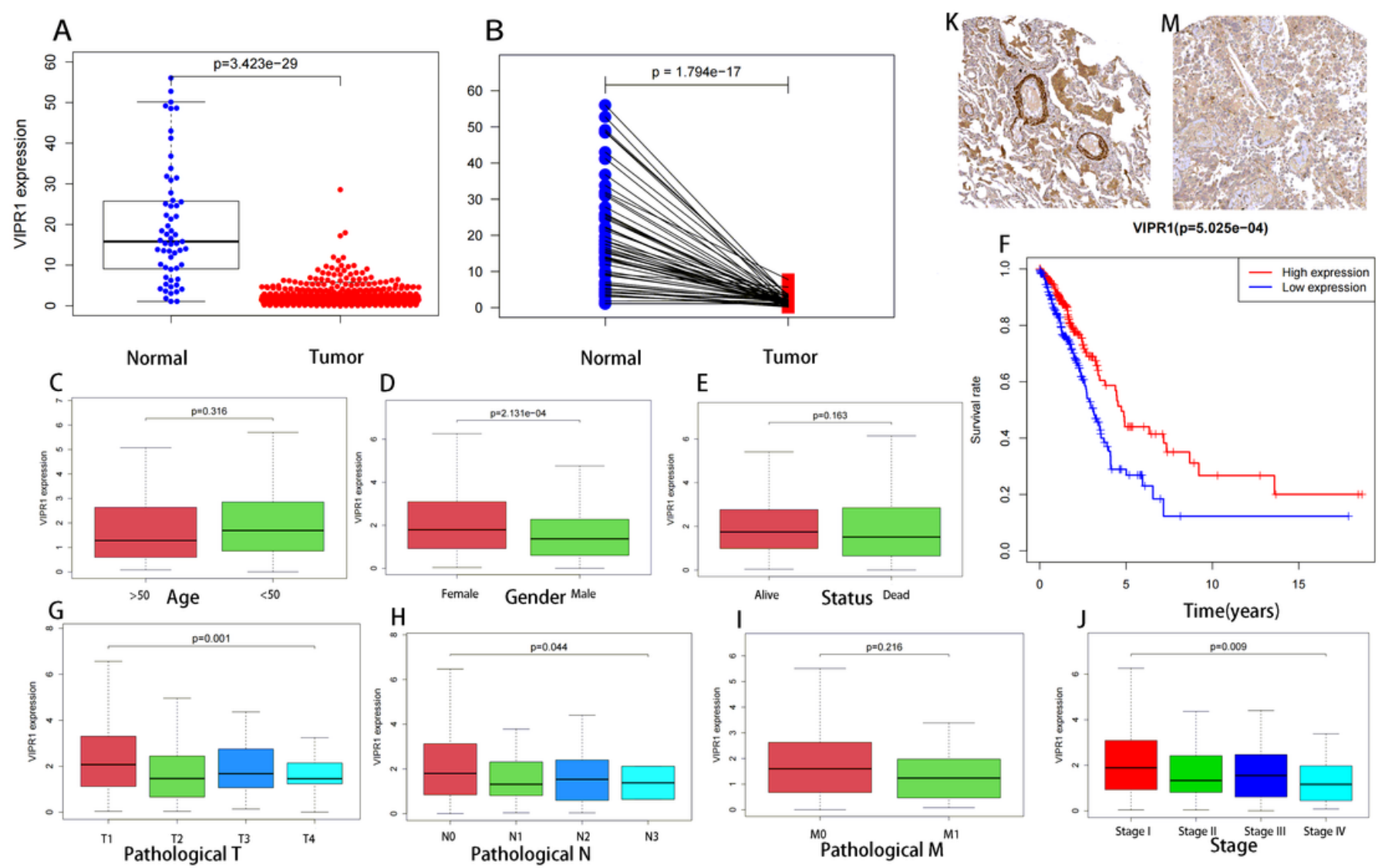

\section{Figure 1}

The relationship between VIPR1 expression level and clinical characteristics of lung adenocarcinoma patients 

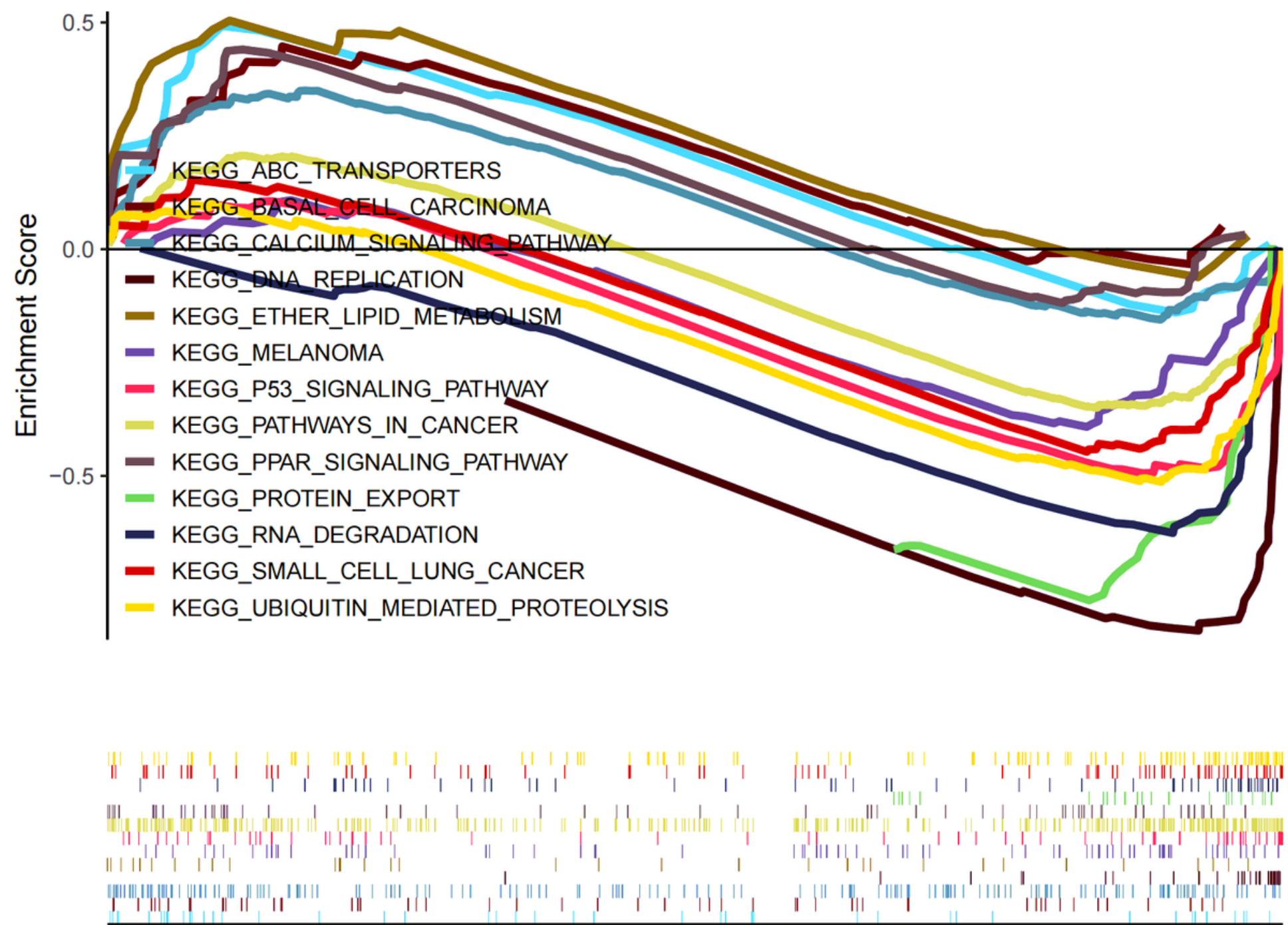

high expression<----------->low expression

Figure 2

VIPR1 functional enrichment analysis 


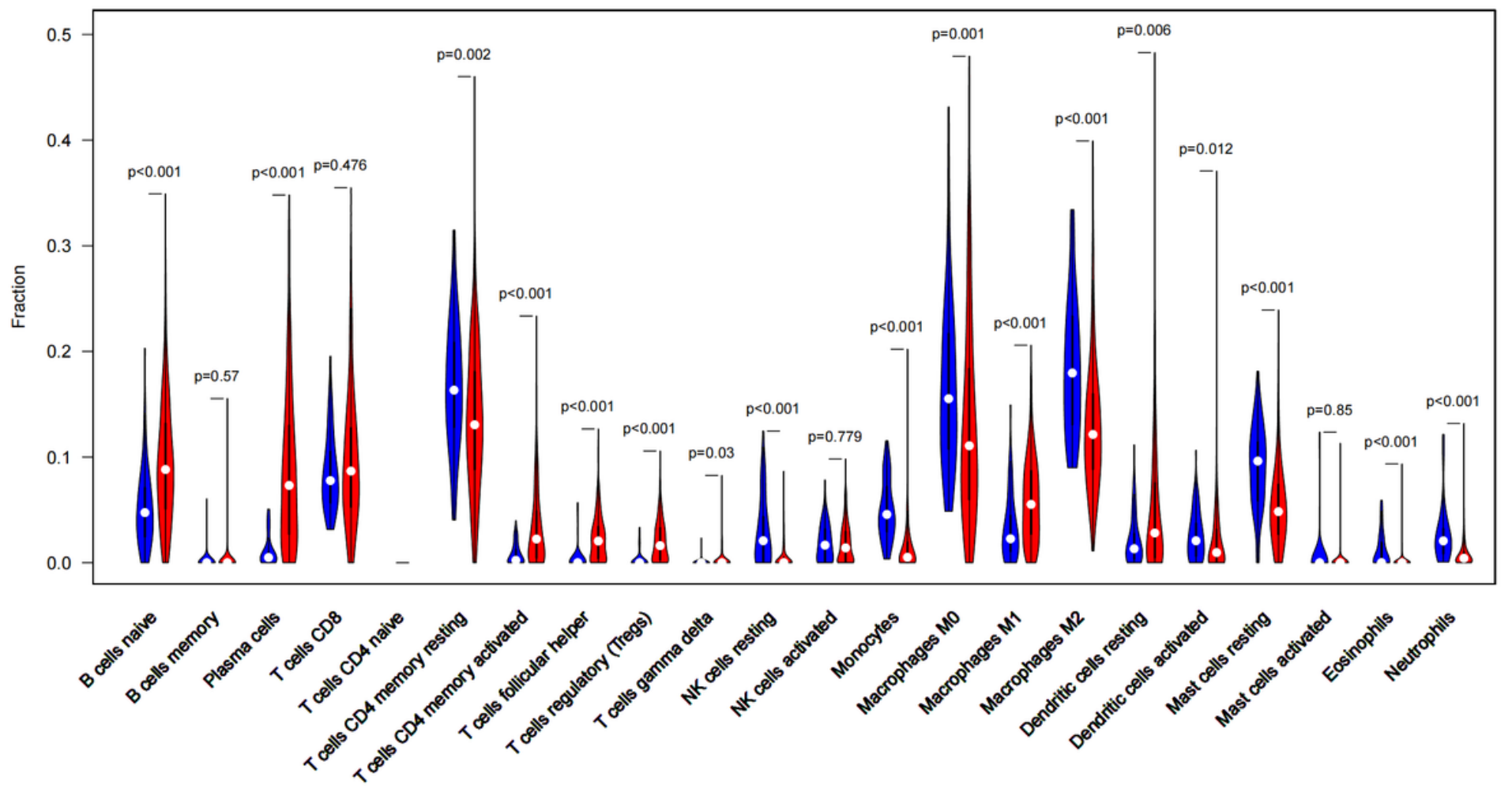

Figure 3

Differences in immune cell composition between normal lung tissue and lung adenocarcinoma tissue 

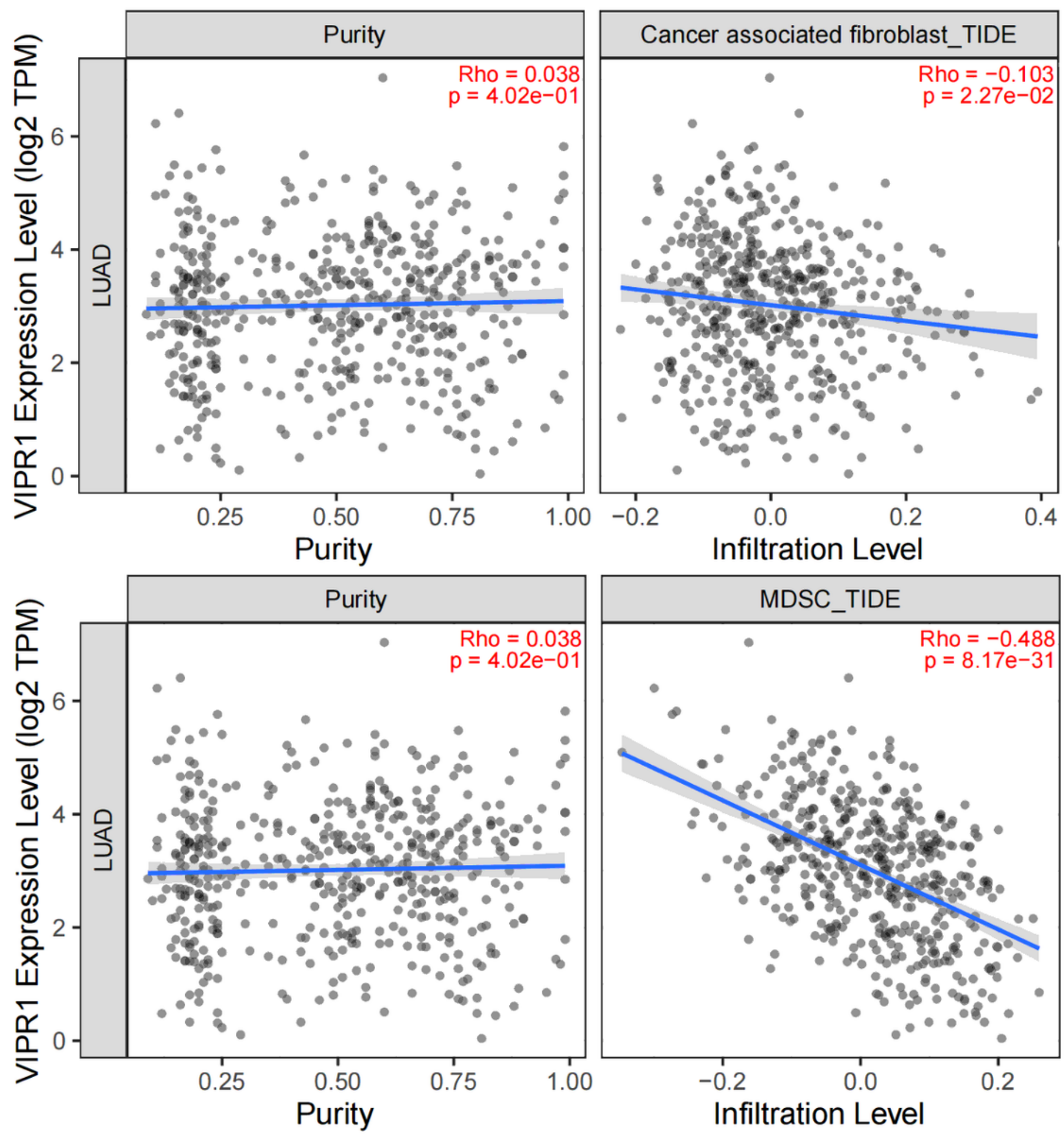

Figure 4

Correlation between VIPR1 expression level and immune infiltration level 